\title{
Covid-19: Deadline for roll out of UK's tracing app will be missed
}

\author{
Stephen Armstrong
}

London

The future of the UK's much vaunted covid-19 contact tracing app seems uncertain after James Brokenshire, the security minister, said on 21 May that he was "unable to give [a] definitive timeline" on when the app might launch.

England's health and social care secretary, Matt Hancock, had previously said that the app, which started trials on the Isle of Wight in early May, would be widely available by mid-May, but the launch has been repeatedly pushed back. At the daily Downing Street briefing on 21 May Hancock acknowledged that a tracing app would not be ready by 1 June, when England is due to enter the next phase of lockdown easing.

John Newton, Public Health England's director of health improvement, told the briefing, "The test and trace programme has more than one component ... which is completely independent of the proximity app. They are distinct but complimentary. And it's perfectly okay, in fact possibly advantageous, to introduce the one before the other."

Contact tracing has been used for decades to control the spread of infectious diseases but was abandoned by the UK government on 12 March when testing capacity was refocused from the community to hospitals.

In mid-April, however, a team from Oxford University showed that a single day's delay in contact tracing could mean the difference between getting the virus under control and a resurgence and suggested that a mobile phone contact tracing app could speed up contact tracing and ease the lockdown. ${ }^{2}$

But doubts about the UK's app have grown as it seems to have faltered during testing on the Isle of Wight. In April the University of Oxford's Big Data Institute predicted that $80 \%$ of current smartphone users would need to use an app to have an effect on the pandemic. ${ }^{3}$ By early May, however, Matthew Gould, chief executive of NHSX, the unit charged with the digital transformation of care, said that $60 \%$ of the population needed to adopt the app to "substantially reduce" the virus spreading, adding that just $20 \%$ uptake would still give "important insights into how the virus is spreading."

So far, the NHSX app has been downloaded around 60000 times, government figures show, although the app can be downloaded outside the test zone, so the true number of people using it on the Isle of Wight, whose total population is around 140000 , is uncertain.

The app's safety and efficiency have been called into question after experts found that personal and health information could be available to cyber-criminals. ${ }^{4}$
There are also doubts over the usefulness of any app. Iceland's Rakning C-19 app has been downloaded by $38 \%$ of Iceland's population of $364000,{ }^{5}$ but Gestur Pálmason, a detective inspector with the Icelandic Police Service who is overseeing contact tracing efforts, described the technology as "more or less ... I wouldn't say useless, but it's the integration of the two [tracing and testing] that gives you results. I would say it has proved useful in a few cases, but it wasn't a game changer."

Eva Blum-Dumontet, senior researcher in charge of healthcare issues at the charity Privacy International, told The BMJ that the very idea of the app was tackling the problem "the wrong way around."

"If we had a system where any person who may be at risk could have access to a test easily, then we can have a conversation about the relevance of an app," she argued. "Instead we have a complex problem. We have the highest death rate in the world, the government doesn't know how to get out of this situation, so they've created an app. This is part of a wider problem that predates covid-19: Matt Hancock relying on technology firms to solve complex issues."

\section{The technology behind tracing apps}

Contact tracing apps for smartphones use wireless technology such as Bluetooth to exchange a "digital handshake" with another user when they come within a set distance of each other for a particular length of time, two metres and 15 minutes in the case of the UK app. This handshake can run in two ways: Apple and Google's "exposure notification API," released this week, which allows phones to inform each other anonymously if their owners have been in contact and one person develops covid-19, or the NHSX method, which uses existing phone software to inform the NHS as well as other smartphone users. NHSX is already working on a second smartphone app, with sources telling The $B M J$ that this alternative app, being made "in parallel," according to NHSX's Matthew Gould, is less reliant on a central database, though it is unclear whether the parallel app involves the new Apple and Google API.

lacobucci G. Covid-19: Lack of capacity led to halting of community testing in March, admits deputy chief medical officer. BMJ 2020;369:m1845. 10.1136/bmj.m1845 32376638 University of Oxford. Digital contact tracing can slow or even stop coronavirus transmission and ease us out of lockdown. Apr 2020. www.ox.ac.uk/news/2020-04-16-digital-contacttracing-can-slow-or-even-stop-coronavirus-transmission-and-ease-us.

3 Kelion L. Coronavirus: NHS contact tracing app to target $80 \%$ of smartphone users. BBC News. Apr 2020. https://www.bbc.co.uk/news/technology-52294896.

4 Culnane C, Teague V. Security analysis of the NHS COVID-19 App. May 2020. https:// www.stateofit.com/UKContactTracing.

5 Directorate of Health of Iceland. Contagion tracing is a community affair https://www.covid. is/app/en.

6 Etherington D. Contagion tracing is a community affair. May 2020. https://techcrunch.com 2020/05/20/apple-and-google-launch-exposure-notification-api-enabling-public-healthauthorities-to-release-apps.

7 UK Govt now building second Covid-19 contact tracing app with Apple and Google. Netimperative. May 2020. www.netimperative.com/2020/05/11/uk-govt-now-buildingsecond-covid-19-contact-tracing-app-with-apple-and-google. 
